\title{
Interactive effects between carbon allotrope fillers on the mechanical reinforcement of polyisoprene based nanocomposites
}

\author{
S. Agnelli ${ }^{1}$, V. Cipolletti ${ }^{2}, S$. Musto $^{2}$, M. Coombs ${ }^{3}$, L. Conzatti ${ }^{4}$, S. Pandini ${ }^{1}$, T. Ricco ${ }^{1}$, \\ M. Galimberti ${ }^{2,5^{*}}$ \\ ${ }^{1}$ Università degli Studi di Brescia, Via Valotti 9, 25123 Brescia, Italy \\ ${ }^{2}$ Politecnico di Milano, Via Mancinelli 7, 20131 Milano, Italy \\ ${ }^{3}$ Pirelli Tyre, Viale Sarca 222, 20126 Milano, Italy \\ ${ }^{4}$ Consiglio Nazionale delle Ricerche, ISMAC UOS Genova, Via De Marini 6, 16149 Genova, Italy \\ ${ }^{5}$ Consiglio Nazionale delle Ricerche, ISMAC, Via E. bassini 15, 20133, Milano, Italy
}

Received 11 November 2013; accepted in revised form 22 February 2014

\begin{abstract}
Interactive effects of carbon allotropes on the mechanical reinforcement of polymer nanocomposites were investigated. Carbon nanotubes (CNT) and nano-graphite with high shape anisotropy (nanoG) were melt blended with poly(1,4cis-isoprene), as the only fillers or in combination with carbon black (CB), measuring the shear modulus at low strain amplitudes for peroxide crosslinked composites. The nanofiller was found to increase the low amplitude storage modulus of the matrix, with or without $\mathrm{CB}$, by a factor depending on nanofiller type and content. This factor, fingerprint of the nanofiller, was higher for CNT than for nanoG. The filler-polymer interfacial area was able to correlate modulus data of composites with CNT, CB and with the hybrid filler system, leading to the construction of a common master curve.
\end{abstract}

Keywords: nanomaterials, carbon nanotubes, nanographite, carbon black, interactive effects

\section{Introduction}

Nanofillers have a great effect on polymer properties [1,2], as the nano-size of such particles implies a high surface area that leads to a high interfacial area with the polymer and to the formation of filler networks at very low nanofiller concentrations.

Nowadays, most investigated nanofillers are carbon nanotubes (CNT) [1-4], graphene, a two-dimensional sheet made of $\mathrm{sp}^{2}$-hybridized carbon atoms in an extended honeycomb network [5], and graphitic nanofillers [6-9] made by few layers of graphene (GE), usually indicated as graphite nanoplatelets, graphite nanosheets, graphite nanoflakes or just simply exfoliated or expanded graphite. Reviews are available on polymer nanocomposites (PNC) based on these types of nanofillers [8-11]. In polymer melts and elastomers, the formation of networks at low nanofiller concentration leads to high values of the dynamic modulus at low strain amplitudes, that goes however along with a pronounced reduction as the strain amplitude increases, phenomenon known as Payne effect [12]. Most studies reported in literature refer to PNC made by a single type of carbon nanofiller in a neat polymer matrix. However, an increasing interest is for elastomer based composites with hybrid filler systems, with a carbon nanofiller combined with the so-called nanostructured filler, carbon black (CB). In fact, the use of hybrid filler systems is considered to make it easier a large scale application of nanofillers.

\footnotetext{
${ }^{*}$ Corresponding author, e-mail: maurizio.galimberti@polimi.it (C) BME-PT
} 
Papers have been published on PNC based on CB and either CNT or nano-graphite. In the case of elastomer polymer matrices, synergistic effects were envisaged [4] for CNT-CB system. CB particles were demonstrated to improve CNT dispersion in a styrene-co-butadiene copolymer matrix [13]. CNT were shown to interact with the surface of $\mathrm{CB}$ (N330) and good improvements in mechanical properties and electrical conductivity were reported, with a percolation threshold lower than that obtained with only CNT [13]. For such systems, connected filler structures were observed and commented [14]. In the case of a polyolefin elastomer (EPDM) [15], an enhancement of the mechanical reinforcement was reported to arise even by adding a small amount of CNT (about $3 \mathrm{php}$ ) to a considerable higher content of CB (N550, $40 \mathrm{php}$ ). A very high electrical conductivity was measured, much higher than that due to $\mathrm{CB}$ alone. In acrylonitrile-co-butadiene copolymer [16], it was reported that CNT (up to 9 php) and a conductive CB (20 or $40 \mathrm{php}$ ) induce reinforcing effects on tensile modulus and strength. Papers are as well available with nano-graphite and $\mathrm{CB}$ as the hybrid filler system. In NBR as the matrix, it was found [17] that the coefficient of friction and the specific wear rate were reduced when $\mathrm{CB}$ is associated to the nano-graphite, thanks to the formation of graphite lubricant films. In natural rubber as the polymer matrix (with epoxidized natural rubber as the compatibilizer), improvements of mechanical, thermal and dynamic-mechanical properties were observed [18] when the nanographite was added to CB (N234).

Previous research of the authors on PNC with hybrid carbon fillers in a poly(1,4-cis-isoprene) (PI) matrix revealed a dramatic enhancement of the material initial modulus, when a small content of nanofiller was added to a composite containing a prevailing amount of CB (N326, $60 \mathrm{php})[19,20]$, and also a remarkable reduction of the modulus with the increase of the strain amplitude. In the case of CNT as the nanofiller [19], transmission electron microscopy (TEM) analysis documented the formation of hybrid CNTCB networks at very low CNT content (about 2 php). The initial modulus values of the nanocomposites containing the hybrid CNT-CB filler network were found to be much higher than those calculated through the simple addition of the initial moduli of the composites containing only $\mathrm{CB}$ and only CNTs, and synergism between the two fillers was thus commented [19]. In ref. [20], CB was used in combination with a nano-graphite with a high shape anisotropy [21] (hereinafter nanoG), defined as the ratio between the crystallites dimensions in directions orthogonal and parallel to structural layers. Hybrid nanoG-CB networks were observed in TEM micrographs from a nanoG level of about 8 php and synergistic effects between nanoG and CB were observed on the initial moduli values of the nanocomposites containing the hybrid filler network.

It can be thus concluded that most prior art indicates a favourable interaction between two carbon allotropes, as it could be expected. In fact, also studies on zeta potential, a measure of the repulsion, in a dispersion, between similarly charged particles, lead to hypothesize an intimate interaction of CB and a nanofiller [22-24].

In the light of these findings and taking into consideration that experiments reported in the literature do not allow an overall rationalization, as data were collected with different types of carbon allotropes at different concentrations in the polymer matrix, it appeared to us worthwhile to perform a more systematic investigation of the interaction between $\mathrm{CB}$ and either CNT or nanoG. The aim was to understand the mechanisms that promote filler network formation and thus the stiffness of the material but also the pronounced non linearity of the dynamicmechanical properties for polymer melts and elastomers. It is worth underlining that such non linearity means dissipation of energy. For example, this hinders nowadays a large scale application of carbon nanofillers in elastomeric materials. A series of composites were prepared in PI as the polymer matrix: with $\mathrm{CB}, \mathrm{CNT}$, nanoG as the only filler or combining $\mathrm{CB}$ with either CNT or nanoG. Structure of nanocomposites was investigated by means of TEM and X-ray diffraction (XRD) analysis. Dynamic storage modulus at low strain amplitude $\left(G_{\gamma \text { min }}^{\prime}\right)$ was determined through dynamic-mechanical measurements in the torsion mode. The experimental values were elaborated according to models available in literature. The results were analyzed in the light of findings from TEM and XRD analyses.

\section{Experimental}

\subsection{Materials}

Synthetic poly(1,4-cis-isoprene) (PI) was SKI3 from Nizhnekamskneftechim Export, with 70 Mooney Units (MU) as Mooney viscosity $\left(M_{\mathrm{L}}(1+4) 100^{\circ} \mathrm{C}\right)$. 
Table 1. Measured BET surface area and DBP absorption number for carbon fillers

\begin{tabular}{|l|c|c|}
\hline Carbon filler & $\begin{array}{c}\text { BET surface area } \\
{\left[\mathbf{m}^{\mathbf{2}} / \mathbf{g}\right]}\end{array}$ & $\begin{array}{c}\text { DBP absorption number } \\
{[\mathbf{m L} / \mathbf{1 0 0} \mathbf{~ g}]^{\mathbf{a}}}\end{array}$ \\
\hline CB & 77.0 & 85.0 \\
\hline CNT & 199.9 & 316.3 \\
\hline nanoG & 330.3 & 162.4 \\
\hline
\end{tabular}

${ }^{\mathrm{a}} \mathrm{mL}$ of absorbed $\mathrm{DBP} / 100$ grams of $\mathrm{CB}$

Carbon Black N326 (CB) was from Cabot, with the following characterization data: $30 \mathrm{~nm}$ as mean diameter of spherical primary particles, nitrogen adsorption number of $77 \mathrm{~m}^{2} / \mathrm{g}$ and DBP adsorption number of $85 \mathrm{~mL} / 100 \mathrm{~g}$.

Multiwall Carbon Nanotubes were Baytubes ${ }^{\circledR} \mathrm{C} 150 \mathrm{P}$ from Bayer Material Science, with a chemical purity $\geq 95$ as $\mathrm{wt} \%$, a length in the $1-10 \mu \mathrm{m}$ range, a number of walls between 3 and 15 and outer and inner diameters of $10-16 \mathrm{~nm}$ and $4 \mathrm{~nm}$ respectively.

NanoGraphite (nanoG) was Synthetic Graphite $8427^{\circledR}$ from Asbury Graphite Mills Inc. In the technical data sheet, the carbon content and the surface area are reported to be at least $99 \mathrm{wt} \%$ and $330 \mathrm{~m}^{2} / \mathrm{g}$, respectively. Chemical composition determined from elemental analysis was, as wt $\%$ : carbon 99.5 , hydrogen 0.4 , nitrogen 0.1 , oxygen 0.0 .

To allow a direct comparison among the three fillers, BET surface areas and DBP absorption numbers were determined with ASTM D6556 and ASTM D2414 methods, respectively. Values are reported in Table 1.

\subsection{Preparation of composites}

Composites were prepared using a Brabender ${ }^{\circledR}$ type internal mixer, with $50 \mathrm{~mL}$ mixing room. Formulations of samples containing only one filler, either $\mathrm{CB}$ or CNT or nanoG, are in Table 2. The same filler vol- ume fractions were used for all the fillers. Samples are labelled CNT, nanoG, $\mathrm{CB}$, with reference to fillers, with a number indicating the filler content, expressed in parts per hundred polymer (php). Formulations of samples containing hybrid filler systems, CB-CNT or CB-nanoG, are in Table 2. They are labelled CB-CNT or CB-nanoG, with reference to the fillers, with a number indicating the $\mathrm{CB}$ content. $\mathrm{CB}$ and the nanofiller have the same volume fraction in the composite.

The following procedure was adopted for the preparation of all the samples: $50 \mathrm{~g}$ of PI were introduced in the Brabender type internal mixer and masticated at $90^{\circ} \mathrm{C}$ for $1 \mathrm{~min}$ with rotors rotating at $30 \mathrm{rpm}$. The filler was then added, mixing was performed for $4 \mathrm{~min}$ and the composite was then discharged at a temperature of about $100^{\circ} \mathrm{C}$. The composite, so prepared, was left to reach room temperature and was fed again to the Brabender mixer kept at a temperature of about $50^{\circ} \mathrm{C}$. Peroxide was added and the final composite was discharged after 2 minutes. Composites were finally further homogeneized by passing them 5 times through a two roll mill operating at $50^{\circ} \mathrm{C}$, with the front roll rotating at $30 \mathrm{rpm}$ and the back roll rotating at $38 \mathrm{rpm}$ and $1 \mathrm{~cm}$ as the nip between the rolls.

\subsection{Characterization}

Crosslinking reaction was studied at $150^{\circ} \mathrm{C}$ with a Monsanto oscillating disc rheometer (MDR 2000) (Alpha Technologies, Swindon, UK), determining the minimum modulus $M_{\mathrm{L}}$, the maximum modulus $M_{\mathrm{H}}$, the modulus $M_{\text {final }}$ at the end of the crosslinking reaction, the time $t_{\mathrm{s} 1}$ required to have a torque equal to $M_{\mathrm{L}}+1$, the time $t_{90}$ required to achieve $90 \%$ of the maximum modulus $M_{\mathrm{H}}$ (i.e. to achieve the optimum

Table 2. Formulations of composites with different fillers ${ }^{\mathrm{a}, \mathrm{b}}$

\begin{tabular}{|l|c|c|c|c|c|c|}
\hline & CB-0 & CB-2.5 & CB-5 & CB-10 & CB-20 & CB-30 \\
\hline CB & 0 & 2.50 & 5.00 & 10.00 & 20.00 & 30.00 \\
\hline & CNT-0 & CNT-2.5 & CNT-5 & CNT-10 & CNT-20 & CNT-30 \\
\hline CNT & 0 & 2.50 & 5.00 & 10.00 & 20.00 & 30.00 \\
\hline & NanoG-0 & NanoG-3 & NanoG-6 & NanoG-11 & NanoG-22 & NanoG-33 \\
\hline NanoG & 0 & 2.78 & 5.56 & 11.11 & 22.22 & 33.33 \\
\hline CB & CB-0-CNT & CB-1.25-CNT & CB-2.5-CNT & CB-5-CNT & CB-10-CNT & CB-15-CNT \\
\hline CNT & 0 & 1.25 & 2.50 & 5.00 & 10.00 & 15.00 \\
\hline & 0 & 1.25 & 2.50 & 5.00 & 10.00 & 15.00 \\
\hline CB & CB-0-NanoG & CB-1.25-NanoG & CB-2.5-NanoG & CB-5-NanoG & CB-10-NanoG & CB-15-NanoG \\
\hline NanoG & 0 & 1.25 & 2.50 & 5.00 & 10.00 & 15.00 \\
\hline
\end{tabular}

${ }^{a}$ Labels and amount of ingredients (expressed in php) are indicated

${ }^{b}$ Other ingredient: PI 100 php, DCUP 1.4 php 
of crosslinking) and the so called reversion, i.e. the relative decrease of the modulus at the end of the crosslinking reaction: $\left(M_{\mathrm{H}}-M_{\text {final }}\right) /\left(M_{\mathrm{H}}-M_{\mathrm{L}}\right) \cdot 100$. TEM analysis was performed with a Zeiss EM 900 microscope applying an accelerating voltage of $80 \mathrm{kV}$. Ultrathin sections (about $50 \mathrm{~nm}$ thick) were obtained by using a Leica EM FCS cryoultramicrotome equipped with a diamond knife (sample temperature: $\left.-130^{\circ} \mathrm{C}\right)$.

Wide-angle X-ray diffraction (WAXD) patterns were taken with an automatic Bruker D8 Advance diffractometer, in reflection, with nickel filtered $\mathrm{Cu}-\mathrm{K} \alpha$ radiation $(1.5418 \AA$ ), at $35 \mathrm{kV}$ and $40 \mathrm{~mA}$. The intensities of reflections in WAXD patterns reported in this manuscript were not corrected for polarization and Lorentz factors, to allow a better visibility of the $(00 \ell)$ peaks. d-spacings were calculated using Bragg's law. The $D_{\mathrm{hk} \ell}$ correlation length of CNT and nanoG crystals was determined applying the Scherrer equation (Equation (1)):

$D_{\mathrm{hk} \ell}=\frac{K \lambda}{\beta_{\mathrm{hk} \ell} \cos \theta_{\mathrm{hk} \ell}}$

where $K$ is the Scherrer constant, $\lambda$ is the wavelength of the irradiating beam $(1.5419 \AA, \mathrm{CuK} \alpha), \beta_{\mathrm{hk} \ell}$ is the width at half height, and $\theta_{\text {hk } \ell}$ is the diffraction angle. The introduction of a correction factor has to be used in case $\beta_{\mathrm{hk} \ell}$ is lower than $1^{\circ}$.

Dynamic-mechanical measurements on crosslinked composites were performed with a Monsanto R.P.A. 2000 rheometer in the torsion mode. For each sample, a first strain sweep $(0.1-25 \%$ shear strain amplitude) was performed at $50^{\circ} \mathrm{C}$ and $1 \mathrm{~Hz}$, then the sample was kept in the instrument at the minimum strain amplitude $\left(\gamma_{\min }=0.1 \%\right)$ for $10 \mathrm{~min}$, to achieve fully equilibrated conditions. Finally, dynamic tests were performed at $50^{\circ} \mathrm{C}$ at increasing strain amplitude $(0.1-25 \%$ shear strain amplitude) with a frequency of $1 \mathrm{~Hz}$.

\section{Results and discussion}

\subsection{Electron microscopic analysis}

Composite structure was investigated by electron microscopy, analyzing samples with the highest filler contents.

Figure 1 shows representative images of composites with the highest content of each carbon allotrope: CNT-30 (Figure 1a), nanoG-33 (Figure 1b) and CB-30 (Figure 1c).
Micrograph in Figure 1a, taken at high magnification, shows an even distribution of isolated and entangled CNT tubes that create a continuous network throughout the PI matrix. As previously observed [19], the melt blending of CNT with the PI matrix leads to a remarkable shortening of the original CNT length, that is reported to be up to $10 \mu \mathrm{m}$ by the supplier. In Figure 1b, agglomerates of nano-G are shown in nanoG-33 sample: they are not larger than $10 \mu \mathrm{m}$ and most of them have submicrometric size, with a disordered structure of the graphitic layers. The presence of single graphite layers and stacks of few of them indicates a high level of delamination. Nanofiller particles appear to be evenly distributed, giving rise to almost continuous network. In Figure 1c, fine and sub-micrometric aggregates of $\mathrm{CB}$ appear to give rise to networks, though not continuous.

Figure 2 shows representative TEM micrographs of composites with the highest contents of the hybrid filler systems: CB-15-CNT (Figure 2a) and CB-15nanoG (Figure 2b). A continuous hybrid CNT-CB network is clearly visible at high magnification in Figure 2a. CNTs lie very close to CB aggregates, thanks to the good interaction between the two carbon allotropes. The high aspect ratio of CNT tubes and their ability to wrap around CB aggregates appear to play a key role in creating the hybrid network.

Distribution and dispersion of nanoG appear to be improved by the presence of CB. In fact, in Figure $2 b$, nanoG agglomerates appear smaller than those present in TEM micrographs of Figure 1b, with a larger amount of single graphite layers or of stacks of few of them. However, no continuous hybrid filler network is observed even in the sample containing a total amount of filler above the percolation threshold of nanoG and close to the percolation threshold of CB. NanoG tends to remain stacked and, in most cases, appears preferentially adhered to the $\mathrm{CB}$ particles.

\subsection{Mechanical characterization and data elaboration}

The mechanical behavior of the crosslinked composites based on PI filled with only one carbon allotrope (either CB or CNT or nanoG; formulations are in Table 2) or with binary filler systems (formulations are in Table 2) was studied by means of dynamic-mechanical tests. For each composite, the 


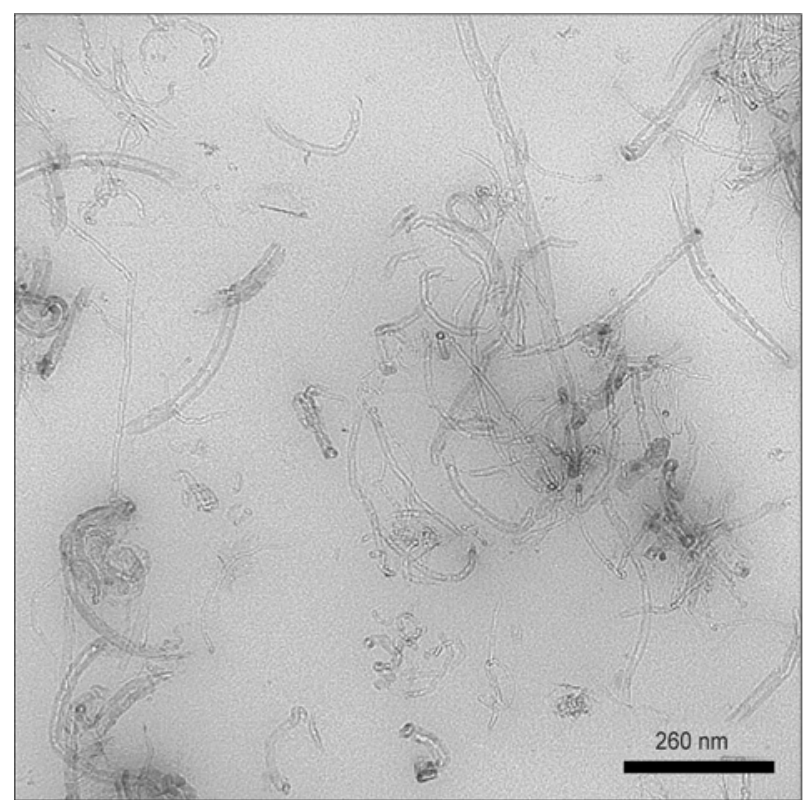

a)

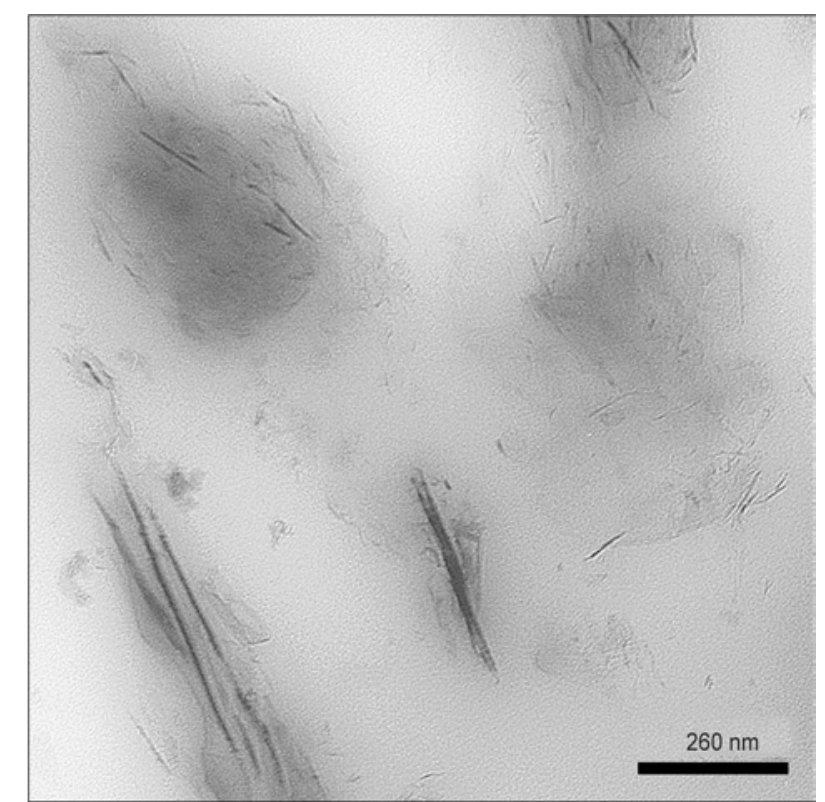

b)

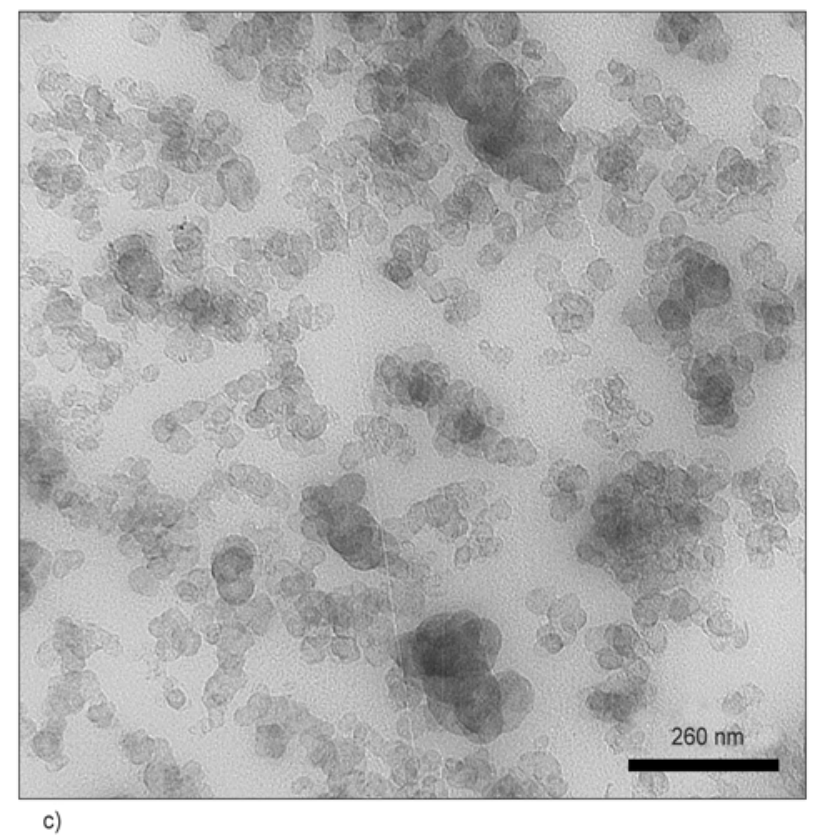

Figure 1. TEM micrographs of CNT-30 (a), nanoG-33 (b) and CB-30 (c)

storage shear modulus $\left(G^{\prime}\right)$ was measured as a function of the strain, determining $G^{\prime}$ values up to $25 \%$ as strain amplitude.

Figure 3 shows an example of $G^{\prime} v s$ shear strain curves evaluated for composites with about $20 \mathrm{php}$ as the overall filler content. Curves of CB filled PI and CNT filled PI are compared with the one of the composite with the hybrid CB-CNT system in Figure $3 \mathrm{a}$ and analogous comparison is shown in Figure $3 \mathrm{~b}$ for composites with $\mathrm{CB}$ and nanoG.

The analysis of the reinforcement of single and binary filler systems was carried out on $G^{\prime}$ values taken at the minimum shear strain amplitude, $G_{\gamma \min }^{\prime}$. Figure 3 clearly highlights the higher reinforcing efficiency of CNT: $G^{\prime}$ for CNT-20 is nearly double that of nanoG-22, and $G^{\prime}$ values of both CNT-20 and nanoG-22 are higher than the $G^{\prime}$ value of CB-20. By replacing half of the nanofiller with the same amount of $\mathrm{CB}, G_{\gamma \min }^{\prime}$ values are reduced and, for both nanofillers, storage moduli of the hybrid composites lie between the values of single filler composites. In Figure $4, G_{\gamma \min }^{\prime}$ values are plotted as a function of the total filler volume fraction, i.e. $\Phi_{\text {nanofiller }}+\Phi_{\mathrm{CB}}$, for all the investigated systems.

Figure 4 clearly shows the typical reinforcing effect of a filler in a molten polymer or in an elastomeric matrix. In fact, it is known that the modulus of the composite is remarkably increased when the added 


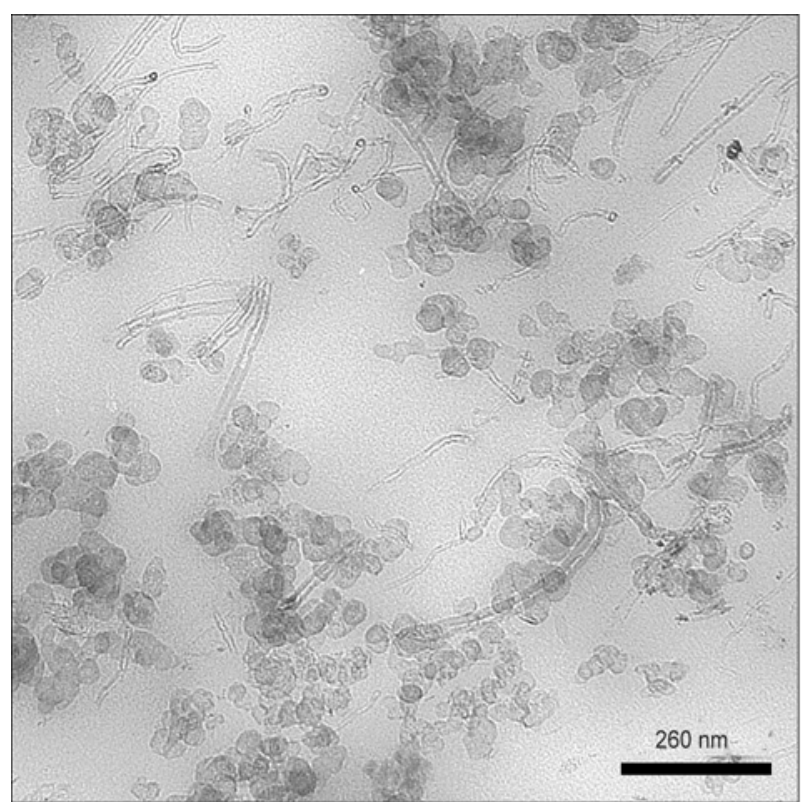

a)

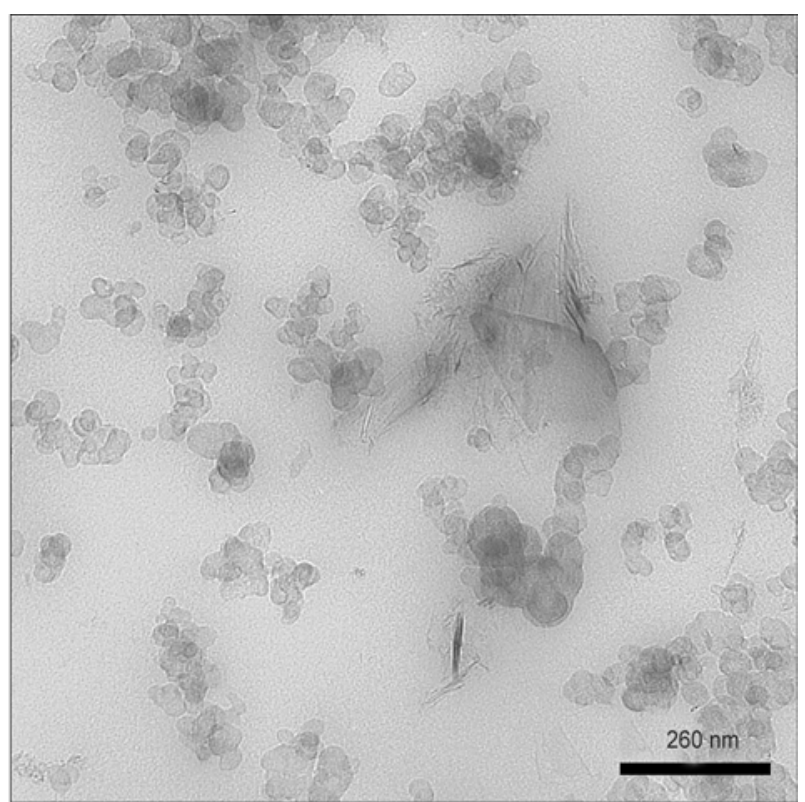

b)

Figure 2. TEM micrographs of CB-15-CNT (a) and CB-15-nanoG (b)

filler(s) have a modulus much higher than that of the matrix. The effect of the reinforcing fillers becomes larger as the filler content increases. To describe such reinforcement, micromechanical models have been developed in the frame of continuum micro- mechanics approach [25-27]. These models attribute the composite reinforcement to the replacement of part of the soft matrix with the stiffer particles, and take into account the non-linear dependence of the modulus on the filler concentration as the result
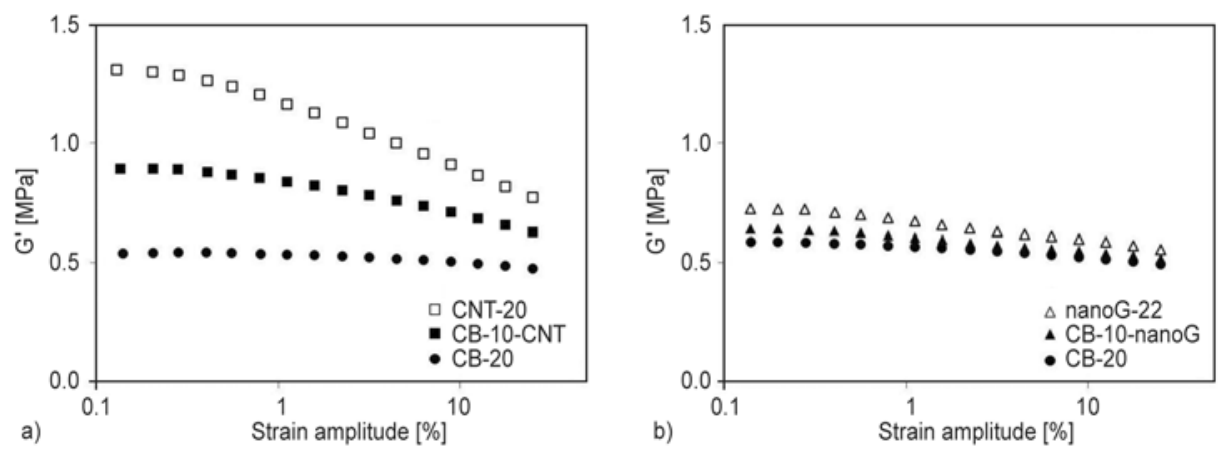

Figure 3. Storage shear modulus $G^{\prime} v s$ strain amplitude for PI filled with 20 php of filler(s): CB and CNT as the fillers (a), $\mathrm{CB}$ and nanoG as the fillers (b)
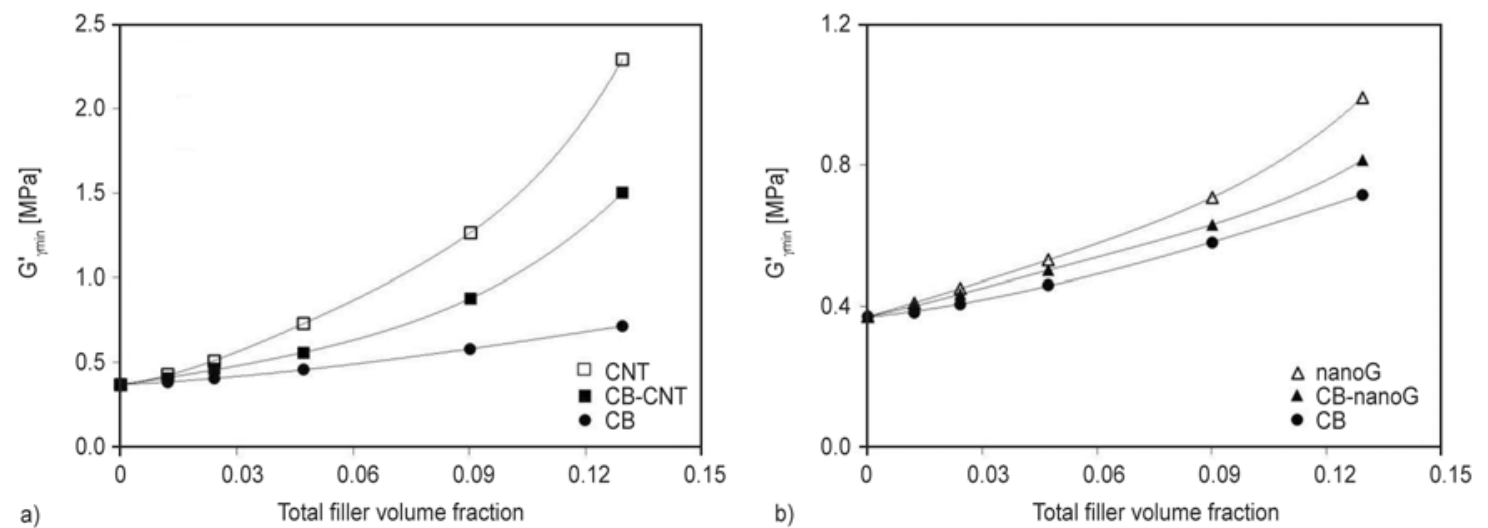

Figure 4. Experimental values of $G_{\gamma \min }^{\prime} v s$ total filler volume fraction (nanofiller:a) CNT, b) nanoG); lines indicate polynomial curves fitting the experimental data 
of interactions of stress fields of each filler particle. The enhancement of the matrix elastic modulus, due to the filler addition, can be expressed for spherical filler particles through the Smallwood-Guth-Gold equation (Equation (2)) [28]:

$$
\frac{G_{\mathrm{c}}}{G_{\mathrm{m}}}=1+2.5 \Phi+14.1 \Phi^{2}
$$

where $G_{\mathrm{c}}$ is the elastic modulus of the composite, $G_{\mathrm{m}}$ is the elastic modulus of the neat elastomer, $\Phi$ is the filler volume fraction and the quadratic term accounts for the mutual disturbance of filler particles. For non spherical particles and for particle aggregates, another equation proposed by Guth can be applied [29, 30] (Equation (3)):

$$
\frac{G_{\mathrm{c}}}{G_{\mathrm{m}}}=1+0.67 f \Phi+1.62 f^{2} \Phi^{2}
$$

where $f$ is a shape factor that takes into account the rod-like shape of filler or filler aggregate and is given by the length to width ratio of particle or aggregate. The authors relied on this model in previous studies on elastomer nanocomposites, to fit the mechanical properties of nanocomposites based on PI and containing either CNT [19] or nanoG [20] as the nanofiller. Best fit approach allowed determining the $f$ aspect ratios, that were calculated to be 22 for CNT and 5 for nanoG. Equation (3) was applied also in other works in the literature for determining the aspect ratio $f$ of CNT: $f$ values in the range from 15 to 20 were reported [31] for composites based on a blend of a poly(1,3-butadiene) and poly[styrene-co(1,3-butadiene)], prepared through dry melt blending. For composites based on poly[styrene-co-(1,3butadiene)], prepared through solution blending, $f$ values were in the range from 40 to 45 [4]. In this work, data available for the single filler composites allowed to calculate the $f$ aspect ratio, reported in Table 3, by applying the best fit approach to the Guth equation (Equation (3)). The $f$ aspect ratio appears to be substantially in line with the one published by some of the authors for nanoG [20], whereas is lower for CNT [19].

Figure 3 shows as well that $G^{\prime}$ decreases with strain amplitude when the composites contain a carbon nanofiller and this decrease is particularly pronounced for composites with CNT as the nanofiller. As mentioned in the Introduction, nanofillers are known to promote a remarkable Payne effect [12]. Several models have been developed in order to explain such an effect on the basis of two main interpretations: the first one, related to the filler networking concept, assumes an agglomeration-deagglomeration process of the filler network above the filler percolation threshold [12, 32-34], the second one, related to filler-matrix interaction, assumes matrix-filler bonding and debonding mechanisms [35-43]. It is not within the scope of this work to discuss the physical mechanisms occurring in the composites. Results will be thus analyzed from a mere phenomenological standpoint, moving from the phenomenon of filler percolation.

In previous author's works on elastomer nanocomposites, initial modulus values of matrix and composites were elaborated to calculate the mechanical percolation of the nanofillers, by means of the Huber-Vilgis plot [44]. The excess of modulus at minimum deformation $\left(G_{\mathrm{c}}-G_{\mathrm{m}}\right) / G_{\mathrm{m}}$, was plotted as a function of the filler volume fraction in a double logarithmic plot. Usually, such plot shows two linear regimes with different slope, at filler contents below and above the percolation threshold, $\Phi_{\mathrm{p}}$. The percolation threshold is therefore evaluated as the filler content at which a discontinuity in the HuberVilgis plot is observed, and it was found to occur at $7.2 \mathrm{php}$ for CNT [19] and at $21.2 \mathrm{php}$ for nanoG [20]. For composites with only one carbon allotrope, $\Phi_{\mathrm{p}}$ values were calculated on the basis of $G_{\gamma \text { min }}^{\prime}$ values, by the Huber-Vilgis plot, and are shown in Table 3. Only the percolation threshold of CB could not be measured in the range of filler contents explored, since it is close to $30 \mathrm{php}$ for N326. Determination of $\Phi_{\mathrm{p}}$ for CB in PI based composites was performed by preparing samples with $\mathrm{CB}$ content higher than $30 \mathrm{php}$ and $\Phi_{\mathrm{p}}$ value was calculated to be $29 \mathrm{php}$, as shown in Table 3. With respect to previous works by the authors $[19,20], \Phi_{\mathrm{p}}$ value is in line for nano$\mathrm{G}$ and is higher for CNT. This latter finding could be justified taking into account the lower CNT aspect ratio, that evidently depends on the mixing conditions experienced by the composite.

When dealing with binary filler systems, the applicability of Guth equation (Equation (3)) decays, and

Table 3. Filler $f$ aspect ratio and percolation threshold, $\Phi_{\mathrm{p}}$, of CB, CNT and nanoG in PI matrix

\begin{tabular}{|l|c|c|}
\hline \multicolumn{1}{|c|}{ Filler } & $\begin{array}{c}\boldsymbol{f} \\
\text { aspect ratio }\end{array}$ & $\begin{array}{c}\boldsymbol{\Phi}_{\mathbf{p}} \\
\text { [php] }\end{array}$ \\
\hline CB & 5 & 29 \\
\hline CNT & 12 & 9 \\
\hline nanoG & 6 & 17 \\
\hline
\end{tabular}


the prediction of the composite modulus by micromechanical models can be very complex. However, it is still possible to evaluate, rather simply, the extent of interaction between two different fillers, highlighting synergistic effects by means of an approach proposed by Sternstein et al. [45], who studied the influence of the mutual interaction of two different fillers on the mechanical reinforcement of an elastomeric matrix. They evaluated interactive effects of nanosilica particles (fumed and surface treated particles to form a binary system) in a poly(vinyl acetate) matrix by calculating the difference between the modulus values of the composite and those predicted by the following simple mixing rule:

$G_{\mathrm{c}}^{\prime}\left(\Phi_{\mathrm{a}}, \Phi_{\mathrm{b}}\right)=G_{\mathrm{a}}^{\prime}\left(\Phi_{\mathrm{a}}\right)+G_{\mathrm{b}}^{\prime}\left(\Phi_{\mathrm{b}}\right)-G_{\mathrm{m}}^{\prime}$

where $G_{\mathrm{c}}^{\prime}\left(\Phi_{\mathrm{a}}, \Phi_{\mathrm{b}}\right)$ is the modulus of a composite with two fillers indicated by $a$ and $b$, with volume fractions $\Phi_{\mathrm{a}}$ and $\Phi_{\mathrm{b}}$, respectively, index c stays for composite, $G_{\mathrm{m}}^{\prime}, G_{\mathrm{a}}^{\prime}\left(\Phi_{\mathrm{a}}\right)$ and $G_{\mathrm{b}}^{\prime}\left(\Phi_{\mathrm{b}}\right)$ are values of modulus of the neat polymer matrix $m$, of the composite with only filler $a$ (volume fraction $\Phi_{a}$ ) and of the composite with only filler $b$ (volume fraction $\Phi_{\mathrm{b}}$ ), respectively. Values from this additive model establish a sort of lower benchmark of expected values and are obtained under the hypothesis that no interaction occurs between two different fillers. In Equation (4), the volume fraction of each filler in the composite with the binary filler system (e.g. $\Phi_{\mathrm{a}}$ of $G_{\mathrm{c}}^{\prime}$ ) is the same as in the composite with only one filler (e.g. $\Phi_{\mathrm{a}}$ of $G_{\mathrm{a}}^{\prime}$ ).

Equation (4) can be rearranged as Equation (5):

$$
\begin{array}{r}
G_{\mathrm{c}}^{\prime}\left(\Phi_{\mathrm{a}}, \Phi_{\mathrm{b}}\right)=G_{\mathrm{m}}^{\prime}+\left(G_{\mathrm{a}}^{\prime}\left(\Phi_{\mathrm{a}}\right)-G_{\mathrm{m}}^{\prime}\right)+\left(G_{\mathrm{b}}^{\prime}\left(\Phi_{\mathrm{b}}\right)-\right. \\
\left.G_{\mathrm{m}}^{\prime}\right)=G_{\mathrm{m}}^{\prime}+\Delta G_{\mathrm{a}}^{\prime}\left(\Phi_{\mathrm{a}}\right)+\Delta G_{\mathrm{b}}^{\prime}\left(\Phi_{\mathrm{b}}\right)
\end{array}
$$

In this work, such model will be referred to as 'additive model', because the composite modulus is simply obtained as the sum of different contributions: the neat matrix modulus and the enhancements of modulus independently produced by each single filler in the same matrix at the same concentration as in the composite. Such model takes into account the nonlinear dependence of initial modulus on each single filler content, by $G_{\mathrm{a}}^{\prime}$ and $G_{\mathrm{b}}^{\prime}$ functions, but it does not include any nonlinearity due to the interactions between two different fillers at $\Phi_{\mathrm{a}}+\Phi_{\mathrm{b}}$ concentration. In the mentioned work [45], the meas- ured values of initial moduli were found to be higher than the ones calculated with Equation (5). The differences between experimental and predicted values were attributed to an interaction term $\left(\Delta G_{\text {int }}^{\prime}\left(\Phi_{\mathrm{a}}, \Phi_{\mathrm{b}}\right)\right)$, that is specific of the binary filler/matrix system, that has to be added to Equation (5) as Equation (6):

$G_{\mathrm{c}}^{\prime}\left(\Phi_{\mathrm{a}, \mathrm{b}}\right)=G_{\mathrm{m}}^{\prime}+\Delta G_{\mathrm{a}}^{\prime}\left(\Phi_{\mathrm{a}}\right)+\Delta G_{\mathrm{b}}^{\prime}\left(\Phi_{\mathrm{b}}\right)+\Delta G_{\mathrm{int}}^{\prime}\left(\Phi_{\mathrm{a}}, \Phi_{\mathrm{b}}\right)$

Such an interaction term is in principle dependent on the fillers concentration (total and relative) as well as on the matrix and on the fillers features (surface area, filler aspect ratio and filler-matrix interaction strength).

Data of composites of the present work, containing only one filler, were examined by using Equation (6). A composite with only one filler can be ideally considered as a binary filler mixture composite, with two parts of the same filler. Evidently, the ratio between said two parts affects the results. In the following discussion, such ratio is fixed to $1: 1$. The total volume fraction $\Phi \varphi$ is thus split in two equal parts $(\Phi / 2)$. If the modulus of the single filler composite could be calculated by applying the additive model, the following equation (Equation (7)), analogous to Equation (5), should give the modulus values:

$$
\begin{aligned}
G_{\mathrm{c}}^{\prime}(\Phi) & =G_{\mathrm{m}}^{\prime}+\Delta G_{\mathrm{c}}^{\prime}\left(\frac{\Phi}{2}\right)+\Delta G_{\mathrm{c}}^{\prime}\left(\frac{\Phi}{2}\right) \\
& =G_{\mathrm{m}}^{\prime}+2 \cdot \Delta G_{\mathrm{c}}^{\prime}\left(\frac{\Phi}{2}\right)
\end{aligned}
$$

Figure 5 shows the $G_{\gamma \min }^{\prime}$ values of composites containing only one filler, as a function of the filler volume fraction. Symbols refer to experimental values and dotted lines indicate values obtained according to the additive model, through Equation (7).

The curves that interpolate the experimental values of the composites modulus (solid lines in Figure 5) diverge from the dotted lines that were drawn on the basis of the additive model, taking values from Equation (7). By examining the curves of Figure 5 in the light of data of Table 3, it appears that the differences between experimental and predicted values for CNT and nanoG become appreciable only when the nanofiller content is above the percolation threshold. In the case of $\mathrm{CB}$, the percolation threshold $\left(\Phi_{\mathrm{p}}\right.$ close to $\left.30 \mathrm{php}\right)$ is only approached and 


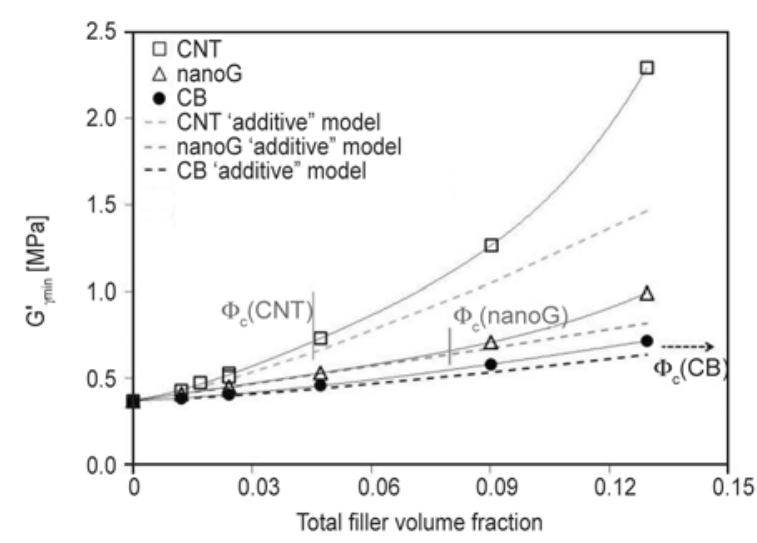

Figure 5. $G_{\gamma \min }^{\prime} v s$ filler volume fraction for $\mathrm{CB}, \mathrm{CNT}$ and nanoG filled composites; broken lines indicate values predicted by the additive model (Equation (7)); the percolation threshold of each system (see Table 6) is also displayed

thus the interactive term is rather small. The excess of modulus could be seen as a sort of 'auto-interactive' term, that can be calculated by means of the following equation (Equation (8)), analogous to Equation (6):

$$
\begin{aligned}
G_{\mathrm{c}}^{\prime}(\Phi) & =G_{\mathrm{m}}^{\prime}+\Delta G_{\mathrm{c}}^{\prime}\left(\frac{\Phi}{2}\right)+\Delta G_{\mathrm{c}}^{\prime}\left(\frac{\Phi}{2}\right) \\
& =G_{\mathrm{m}}^{\prime}+2 \cdot \Delta G_{\mathrm{c}}^{\prime}\left(\frac{\Phi}{2}\right)+\Delta G_{\mathrm{int}}^{\prime}\left(\frac{\Phi}{2}, \frac{\Phi}{2}\right)
\end{aligned}
$$

This 'auto-interactive' term is clearly related to the nonlinearity of the examined composite. In fact, if the modulus values of a composite with only one filler follow the Guth model (Equation (2)), by introducing the Guth expression (Equation (3)) for $G_{\mathrm{c}}^{\prime}(\Phi)$ and $G_{\mathrm{c}}^{\prime}(\Phi / 2)$ into Equation (8), the following relationship is obtained (Equation (9)):

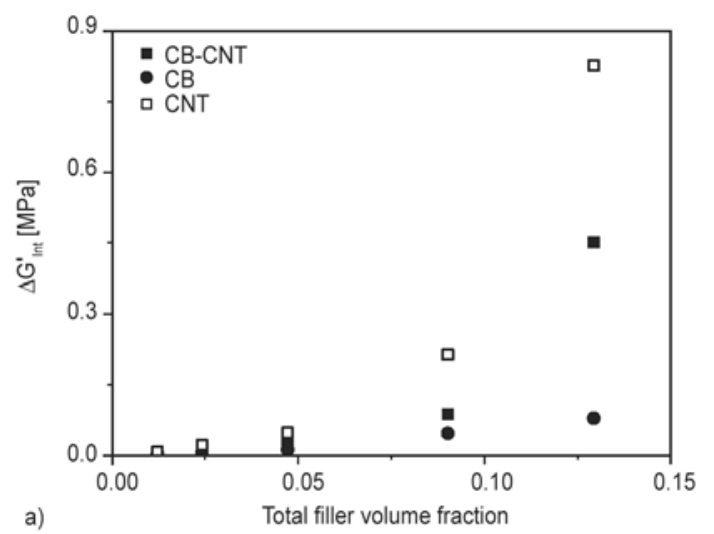

$\Delta G_{\text {int }}^{\prime}\left(\frac{\Phi}{2}, \frac{\Phi}{2}\right)=\frac{1}{2} \cdot 1.62 f^{2} \Phi^{2}$

This equation indicates that the 'auto-interactive' term is related only to the quadratic (nonlinear) term of the dependence of modulus on the filler content. This so called 'auto-interactive' term $\Delta G^{\prime}$ int $(\Phi / 2, \Phi / 2)$ was calculated through Equation (8) for all the composites containing only one filler. Moreover, the interactive term was calculated by applying Equation (6) for composites containing the hybrid filler systems. For this evaluation, experimental data were fitted by polynomial best fitting curves (lines in Figure 4), in order to compute the modulus values at the exact filler contents necessary to evaluate $\Delta G^{\prime}$ int by Equations (6) and (8).

Figure 6 shows the dependence of both the interactive and 'auto-interactive' terms on the total filler volume fraction. Graphs were prepared in order to emphasize the comparison between interactive terms arising from systems with only one filler or with the hybrid filler system.

Some comments can be added. Interactive terms for a given filler mixture (either made by two different fillers or by two parts of the same filler) strongly depend on the filler content, as expected: they are negligible at low contents, increase with the filler amount and appear to be remarkable only at the highest contents. It is definitely worth underlining the more pronounced nonlinearity of composites based on CNT with respect to composites based on nanoG and $\mathrm{CB}$. Most interesting results seem to arise from the comparison of the composites with the hybrid filler systems CB-CNT and CB-nanoG. In fact, while

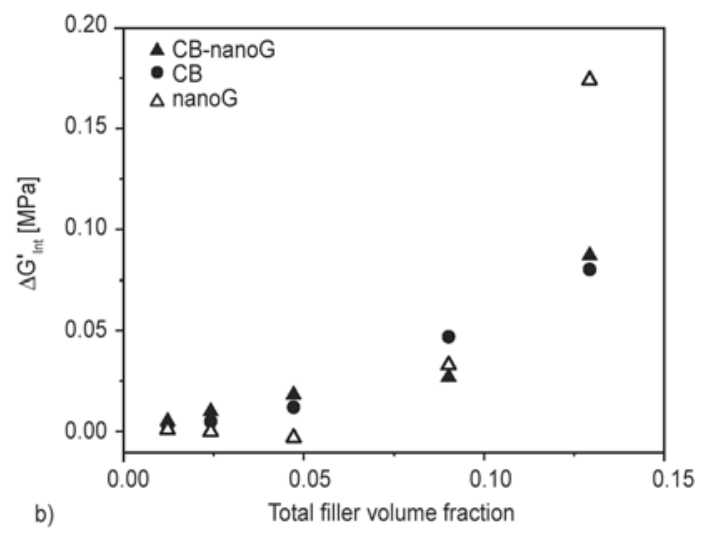

Figure 6. Interactive and 'auto-interactive terms', computed by Equation (6) and (8), respectively, as a function of the total filler volume fraction for the investigated systems; a) CB and/or CNT filled systems, b) CB and/or nanoG filled systems 
values of $\Delta G^{\prime}$ int for the hybrid CB-CNT system (symbols - in Figure 6a) are between those of composites with either only $\mathrm{CNT}$ or only $\mathrm{CB}$, values of $\Delta G^{\prime}$ int for the hybrid CB-nanoG system (symbols in Figure $6 b$ ) are overlapped with the values of the system containing only $\mathrm{CB}$.

Modulus reinforcement is often evaluated as the ratio of the composite modulus over the matrix modulus. In a previous work by some of the authors, the reinforcement of PI filled with only CNT or filled with a mix of CNT and 60 php of CB [19] was evaluated. It was found that the relative increase of modulus brought about by CNT was the same, in the absence and in the presence of CB. $G_{\gamma \text { min }}^{\prime}$ experimental data are in this work elaborated as done in ref. [19]. The relative modulus enhancement is expressed by a $k$ factor, defined as follows: the moduli of composites with only CNT or only nanoG ( $\left.G_{\text {nanofiller }}^{\prime}\right)$ are normalized over the modulus of the neat PI matrix $\left(G_{\mathrm{m}}^{\prime}\right)($ Equation $(10))$ :

$k=\frac{G_{\text {nanofiller }}^{\prime}\left(\Phi_{\text {nanofiller }}\right)}{G_{\mathrm{m}}^{\prime}}$

whereas the moduli of the hybrid filler composites $\left(G_{\mathrm{c}}^{\prime}\right)$ are normalized over the modulus of the composite with only $\mathrm{CB}\left(G^{\prime}{ }_{\mathrm{CB}}\right)$ (Equation (11)):

$k=\frac{G_{\mathrm{c}}^{\prime}\left(\Phi_{\text {nanofiller, }}, \Phi_{\mathrm{CB}}\right)}{G_{\mathrm{CB}}^{\prime}\left(\Phi_{\mathrm{CB}}\right)}$

Figure 7 shows the $k$ factor of the investigated composites, as a function of the nanofiller volume fraction. It is interesting to note that, in agreement with the results shown in ref. [19], in the investigated range of filler content, nanofillers enhance the matrix modulus by a multiplication factor, $k$, that depends only on the nanofiller type and content, no matter if

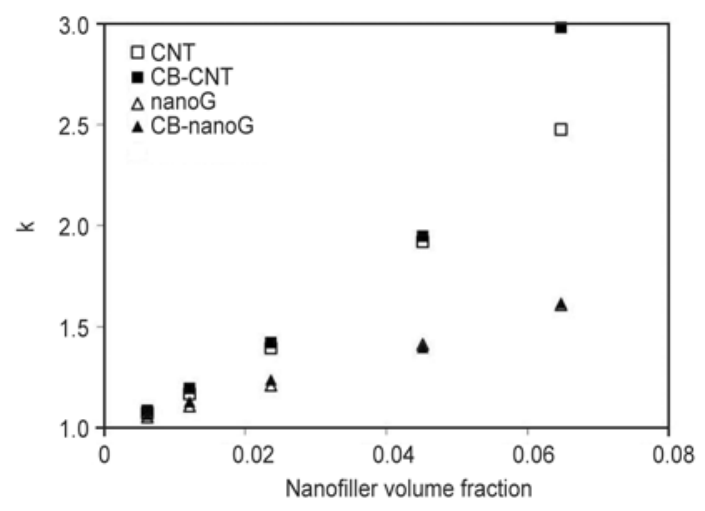

Figure 7. $k$ factor vs nanofiller volume fraction; open symbols: single nanofiller compounds, full symbols: hybrid compounds (nanofiller-CB) the matrix is a neat or a $\mathrm{CB}$ filled polymer. The only exception to this finding are the data of the systems with a CNT content of $6.5 \%$ (about 15 php), which deserve further investigation.

In a recent work [46], the multiplication factor $k$ was calculated for CNT in PI based composites containing 12 php of CNT and 0, 20, 40, 60 php of CB and was found to be in a very narrow range. In particular, by keeping the same volume fraction of CNT in composites containing either 0 or 60 php of $\mathrm{CB}, k$ was observed to be 3.2 and 3.4, respectively.

On the basis of the observation that the multiplication factor, $k$, does not depend on the composition of the matrix (that can be either neat or filled with $\mathrm{CB}$ ), the Equation (12) can be written:

$$
\begin{aligned}
k\left(\Phi_{\text {nanofiller }}\right) & =\frac{G_{\text {nanofiller }}^{\prime}\left(\Phi_{\text {nanofiller }}\right)}{G_{\mathrm{m}}^{\prime}} \\
& =\frac{G_{\mathrm{c}}^{\prime}\left(\Phi_{\text {nanofiller }}, \Phi_{\mathrm{CB}}\right)}{G_{\mathrm{CB}}^{\prime}\left(\Phi_{\mathrm{CB}}\right)}
\end{aligned}
$$

with $k\left(\Phi_{\text {nanofiller }}\right)$ independent of $\Phi_{\mathrm{CB}}$.

By combining Equation (12) with Equation (6), this Equation (13) was derived:

$k\left(\Phi_{\text {nanofiller }}\right)=1+\frac{\Delta G_{\text {int }}^{\prime}\left(\Phi_{\text {nanofiller }}, \Phi_{\mathrm{CB}}\right)}{\left(G_{\mathrm{CB}}^{\prime}\left(\Phi_{\mathrm{CB}}\right)-G_{\mathrm{m}}^{\prime}\right)}$

Equation (13) shows that $k>1$ implies that $\Delta G^{\prime}{ }_{\text {int }}\left(\Phi_{\text {nanofiller, }}, \Phi_{\mathrm{CB}}\right)$ is $\neq 0$, that means there is interaction between the nanofiller and CB, what was named above as synergy. As a matter of fact, Equation (12) itself reveals the interaction between the two different fillers. In fact, Equation (12) shows that the modulus of the composite $G^{\prime}$ with both fillers can be obtained by multiplying the moduli of composites containing only one filler, as shown by Equation (14):

$$
G_{\mathrm{c}}^{\prime}\left(\Phi_{\text {nanofiller }}, \Phi_{\mathrm{CB}}\right)=\frac{G_{\text {nanofiller }}^{\prime}\left(\Phi_{\text {nanofiller }}\right) \cdot G_{\mathrm{CB}}^{\prime}\left(\Phi_{\mathrm{CB}}\right)}{G_{\mathrm{m}}^{\prime}}
$$

These comments concerning $k$ factor are based on the collected experimental data and a more general validity (for other filler systems) has still to be demonstrated.

Figure 1 clearly shows that $k$ of CNT is higher than $k$ of nanoG. The higher value of the $k$ factor for CNT with respect to nanoG could be considered in line with what shown in the TEM micrographs: CNT does 

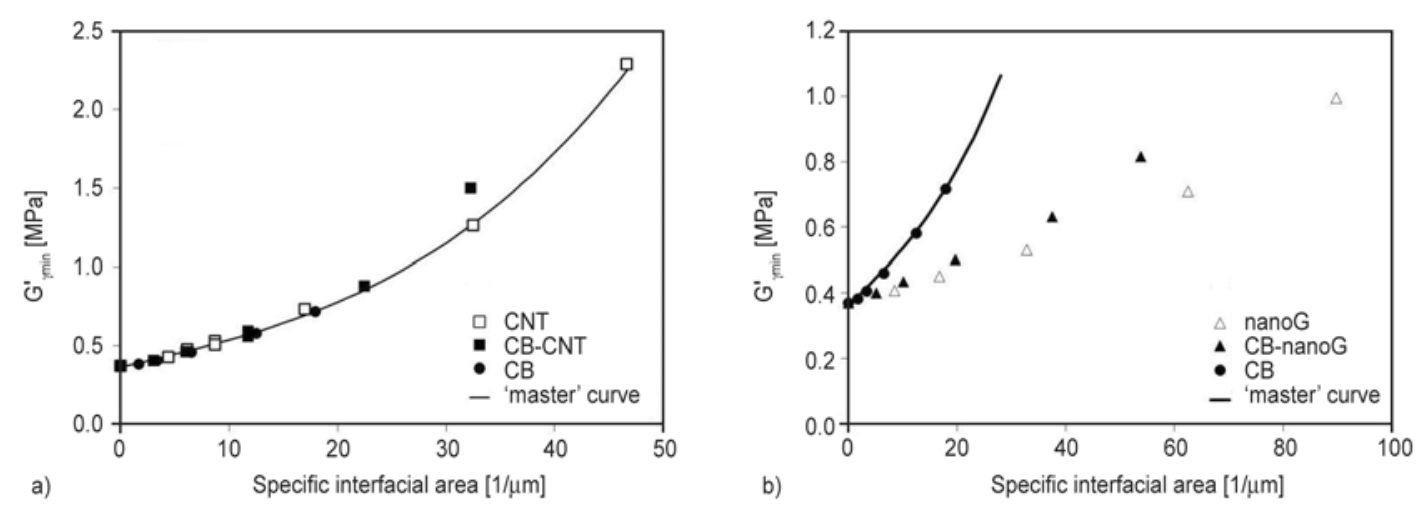

Figure 8. $G_{\gamma \min }^{\prime}$ values as a function of the specific filler - matrix interfacial area for the different compounds; the 'master' curve averaging the three fitting curves of the systems in a) is shown as well

have a higher ability to form networks, with itself and with a nano-structured filler such as CB. However, the high content of nanoG required to build networks is to an extent unexpected, on the basis of BET surface area data reported in Table 1.

It appears thus worthwhile to investigate the role played by surface area and, in particular, by fillerpolymer interfacial area in determining the initial modulus of the composite material. Such investigation is more significant in the light of recent literature [34, 40], according to which the matrix properties do not keep constant with filler loading, rather they change depending on the amount of filler-matrix interface area $\left(A_{\mathrm{i}}\right)$. In particular, it was shown [40] that the reinforcement increases by changing particle shape at the same filler volume fraction i.e. by increasing surface area, for composites with the same chemical nature of the fillers.

In Figure $8, G_{\gamma \min }^{\prime}$ data are plotted versus the specific filler - matrix interfacial area (the interfacial area normalized over the composite volume), evaluated as $A_{\mathrm{i}} \cdot \rho \cdot \Phi$, where $A_{\mathrm{i}}$ is assumed to be equal to BET surface area (see Table 1) and $\rho$ is the filler density. For hybrid filler systems, the specific filler - matrix interfacial area is evaluated for each filler at the corresponding volume fraction and the results are simply added to give the overall area. Figure $8 \mathrm{a}$ shows the experimental points for the composites containing only CNT, only $\mathrm{CB}$ and the hybrid CBCNT system. Points referring to the three different composites appear to lie on a common 'master' curve (solid line in both Figure $8 \mathrm{a}$ and $8 \mathrm{~b}$ ), evaluated by averaging the three best fitting curves of each system.

This elaboration indicates that the filler surface and thus the filler-matrix interfacial area are the key fea- tures that affect the initial modulus values of the composites. This comment is simply in line with the basic theory of elastomer reinforcement [26, 27]. However for the first time, a common elaboration seems to be possible for nano- and nano-structured fillers. Figure $8 \mathrm{~b}$ shows the 'master' curve evaluated by data of Figure 8a and experimental points for the composites containing only nanoG, only $\mathrm{CB}$ and the hybrid CB-nanoG systems. It is evident that points arising from nanoG based composites are well below the 'master' curve. To justify this finding, it could be hypothesized that the filler surface area of nanoG is not totally accessible to the polymer chains. In fact, Table 2 shows that, although the BET surface area of nanoG is higher than that of CNT, DBP absorption for nanoG is lower than for CNT. It is known [27] that the DBP absorption is correlated with the volume of the polymer occluded by the filler. Points in Figure $8 \mathrm{~b}$ could be brought on the 'master' curve, by assuming for nanoG a surface area of about $90 \mathrm{~m}^{2} / \mathrm{g}$. It could be thus hypothesized that the surface areas experimentally determined for $\mathrm{CB}$ and CNT are prevailingly accessible to polymer chains, whereas nanoG layers seem to be accessible to the small Helium atoms used for the BET measurement but not to the bulky chains. This leads to hypothesize that nanoG layers are stacked, forming crystalline aggregates. To investigate the aggregation of layer in nanoG and in CNT, XRD analysis was performed on composites CB-15-nanoG and CB-15CNT. Figure 9a shows the XRD pattern of the nanoG based composite and Figure $9 \mathrm{~b}$ shows the XRD pattern of the CNT based composite. By applying the Scherrer equation (see Equation (1)) to the 002 reflection at $26.5^{\circ}$ as $2 \theta$ value in the pattern of CB15 -nanoG, the number of layers stacked in a crys- 


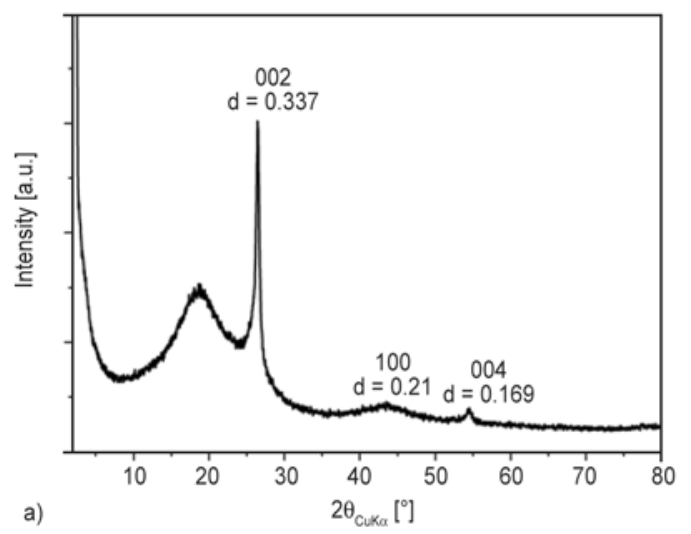

Figure 9. XRD pattern of CB-15-nanoG (a) and CB-15-CNT

talline domain was calculated to be about 72. Interestingly, this number is higher than the one detected in the pristine nanoG sample [21].

It could be commented that the high pressure applied in the crosslinking step brings nanoG to a minimum of energy, that means to a higher crystallinity. This relatively high number of stacked layers confirms that the surface area measured through BET technique is not accessible to the polymer chains and justifies what shown in Figure 8b. By applying the Scherrer equation, the number of layers wrapped to form CNT was calculated to be about 12. Interestingly, also this number is higher than the one found in the pristine sample (10). Also in this case, the crosslinking pressure can be invoked to justify the formation of crystalline domains with a higher correlation length.

\section{Conclusions}

This work is focused on the interactive effects of carbon allotropes on the mechanical reinforcement of a hydrocarbon polymer matrix, poly(1,4-cis-isoprene). So called nano-fillers such as CNT or nano-G are used, in the neat polymer matrix, or combined with a nano-structured filler such as CB.

The reinforcement effects on the shear storage modulus brought about by hybrid fillers in an elastomeric matrix are analysed by a method proposed by Sternstein [45] and quantified by an interaction term. This method relates the extent of filler particles interactions to the nonlinearity of filler reinforcement and allows to assess the ability of the nanofiller to impart such non linearity to the composite with the hybrid filler system thus giving a reliable indication of the filler(s) ability to build networks.

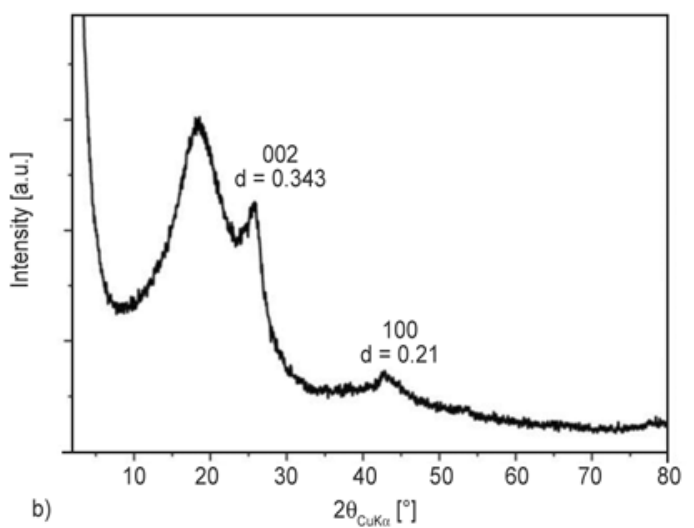

(b) in the $0-80^{\circ}$ range as $2 \theta$ values

CNT were found to have the largest values of the interaction term and to form filler networks, also in presence of $\mathrm{CB}$, at low $\mathrm{CNT}$ concentrations.

A sort of fingerprint is identified for the carbon nanofillers. In fact, the relative increase of modulus, defined as $k$ factor, brought about by a given nanofiller in the pure matrix is the same as in the system with $\mathrm{CB}$. A correlation between the interaction term and $k$ factor is shown.

Finally, the filler-polymer interfacial area is shown to be able to correlate the behaviour of a nano- with a nanostructured filler (CNT with $\mathrm{CB}$ ): experimental storage modulus values lie on the same 'master' curve when plotted versus the specific interfacial area, provided that the surface area is accessible to the polymer. The definition of such a 'master' curve could be seen as a tool to investigate the polymerfiller interfacial area actually available. Moreover, these findings seem to highlight the importance of CNT shape, that allows high values of accessible surface area and the easy formation of networks.

\section{References}

[1] Mittal V.: Advances in polyolefin nanocomposites. CRC Press, Boca Raton (2011).

[2] Maiti M., Bhattacharya M., Bhowmick A. K.: Elastomer nanocomposites. Rubber Chemistry and Technology, 81, 384-469 (2008).

DOI: $10.5254 / 1.3548215$

[3] Moniruzzaman M., Winey K. L.: Polymer nanocomposites containing carbon nanotubes. Macromolecules, 39, 5194-5205 (2006).

DOI: $10.1021 / \mathrm{ma} 060733 \mathrm{p}$

[4] Bokobza L.: Multiwall carbon nanotube elastomeric composites: A review. Polymer, 48, 4907-4920 (2007). DOI: $10.1016 /$ j.polymer.2007.06.046 
[5] Novoselov K. S., Geim A. K., Morozov S. V., Jiang D., Zhang Y., Dubonos S. V., Grigorieva I. V., Firsov A. A.: Electric field effect in atomically thin carbon films. Science, 306, 666-669 (2004). DOI: $10.1126 /$ science. 1102896

[6] Park S., Ruoff R. S.: Chemical methods for the production of graphenes. Nature Nanotechnology, 4, $217-$ 224 (2009).

DOI: $10.1038 /$ nnano.2009.58

[7] Jang B. Z., Zhamu A.: Processing of nanographene platelets (NGPs) and NGP nanocomposites: A review. Journal of Materials Science, 43, 5092-5101 (2008). DOI: $10.1007 / \mathrm{s} 10853-008-2755-2$

[8] Ramanathan T., Stankovich S., Dikin D. A., Liu H., Shen H., Nguyen S. T., Brinson L. C.: Graphitic nanofillers in PMMA nanocomposites - An investigation of particle size and dispersion and their influence on nanocomposite properties. Journal of Polymer Science Part B: Polymer Physics, 45, 2097-2112 (2007). DOI: $10.1002 /$ polb. 21187

[9] Li B., Zhong W-H.: Review on polymer/graphite nanoplatelet nanocomposites. Journal of Materials Science, 46, 5595-5614 (2011). DOI: $10.1007 / \mathrm{s} 10853-011-5572-y$

[10] Potts J. R., Dreyer D. R., Bielawski C. W., Ruoff R. S.: Graphene-based polymer nanocomposites. Polymer, 52, 5-25 (2011). DOI: $10.1016 /$ j.polymer.2010.11.042

[11] Sengupta R., Bhattacharya M., Bandyopadhyay S., Bhowmick A. K.: A review on the mechanical and electrical properties of graphite and modified graphite reinforced polymer composites. Progress in Polymer Science, 36, 638-670 (2011). DOI: 10.1016/j.progpolymsci.2010.11.003

[12] Payne A. R., Whittaker R. E.: Low strain dynamic properties of filled rubbers. Rubber Chemistry and Technology, 44, 440-478 (1971).

DOI: $10.5254 / 1.3547375$

[13] Bokobza L., Rahmani M., Belin C., Bruneel J-L., El Bounia N-E.: Blends of carbon blacks and multiwall carbon nanotubes as reinforcing fillers for hydrocarbon rubbers. Journal of Polymer Science Part B: Polymer Physics, 46, 1939-1951 (2008).

DOI: $10.1002 /$ polb.21529

[14] Bokobza L.: Mechanical, electrical and spectroscopic investigations of carbon nanotube-reinforced elastomers. Vibrational Spectroscopy, 51, 52-59 (2009). DOI: $10.1016 /$ j.vibspec.2008.10.001

[15] Lorenz H., Fritzsche J., Das A., Stöeckelhuber K. W., Jurk R., Heinrich G., Klüppel M.: Advanced elastomer nano-composites based on CNT-hybrid filler systems. Composites Science and Technology, 69, 2135-2143 (2009).

DOI: $10.1016 /$ j.compscitech.2009.05.014
[16] Ryu S-R., Sung J-W., Lee D-J.: Strain-induced crystallization and mechanical properties of NBR composites with carbon nanotube and carbon black. Rubber Chemistry and Technology, 85, 207-218 (2012). DOI: $10.5254 /$ rct.12.88955

[17] Wang L. L., Zhang L. Q., Tian M.: Mechanical and tribological properties of acrylonitrile-butadiene rubber filled with graphite and carbon black. Materials and Design, 39, 450-457 (2012).

DOI: $10.1016 /$ j.matdes.2012.02.051

[18] Malas A., Das C. K., Das A., Heinrich G.: Development of expanded graphite filled natural rubber vulcanizates in presence and absence of carbon black: Mechanical, thermal and morphological properties. Materials and Design, 39, 410-417 (2012).

DOI: $10.1016 /$ j.matdes.2012.03.007

[19] Galimberti M., Coombs M., Riccio P., Riccò T., Passera S., Pandini S., Conzatti L., Ravasio A., Tritto I.: The role of CNTs in promoting hybrid filler networking and synergism with carbon black in the mechanical behavior of filled polyisoprene. Macromolecular Materials and Engineering, 298, 241-251 (2012). DOI: $10.1002 /$ mame. 201200075

[20] Galimberti M., Kumar V., Coombs M., Cipolletti V., Agnelli S., Pandini S., Conzatti L.: Filler networking of a nanographite with a high shape anisotropy and synergism with carbon black in poly(1,4-cis-isoprene)based nanocomposites. Rubber Chemistry and Technology, in press (2014).

DOI: $10.5254 /$ rct. 13.87903

[21] Mauro M., Cipolletti V., Galimberti M., Longo P., Guerra G.: Chemically reduced graphite oxide with improved shape anisotropy. The Journal of Chemical Physics: C, 116, 24809-24813 (2012).

DOI: $10.1021 / j p 307112 \mathrm{k}$

[22] Bhattacharya M., Bhowmick A. K.: Synergy in carbon black-filled natural rubber nanocomposites. Part I: Mechanical, dynamic mechanical properties, and morphology. Journal of Materials Science, 45, 6126-6138 (2010).

DOI: $10.1007 / \mathrm{s} 10853-010-4699-6$

[23] Brochard-Wyart F., de Gennes P. G.: Viscosity at small scales in polymer melts. European Physical Journal E, 1, 93-97 (2000). DOI: $10.1007 / \mathrm{s} 101890050011$

[24] Flores F., Graebling D., Allal A., Guerret-Piécourt C.: Modelization of flow electrification in a polymer melt. Journal of Physics D: Applied Physics, 40, 2911-2919 (2007). DOI: $\underline{10.1088 / 0022-3727 / 40 / 9 / 037}$

[25] Payne A. R.: Dynamic properties of filler-loaded rubbers. in 'Reinforcement of elastomers' (ed.: Kraus G.) Interscience Publishers, New York, 69-114 (1965). 
[26] Donnet J. B., Custodero E.: Reinforcement of elastomers by particulate fillers. in 'The science and technology of rubber' (eds.: Mark J. E., Erman B., Eirich F. R.) Academic Press, San Diego, 367-400 (2005). DOI: 10.1016/B978-012464786-2/50011-0

[27] Medalia A. I.: Effect of carbon black on dynamic properties of rubber vulcanizates. Rubber Chemistry and Technology, 51, 437-523 (1978).

DOI: $10.5254 / 1.3535748$

[28] Guth E., Gold O.: On the hydrodynamic theory of the viscosity of suspensions. Physical Review, 53, 322328 (1938).

[29] Guth E.: Theory of filler reinforcement. Journal of Applied Physics, 16, 20-25 (1945).

DOI: $10.1063 / 1.1707495$

[30] Guth E.: Theory of filler reinforcement. Rubber Chemistry and Technology, 18, 596-604 (1945). DOI: $10.5254 / 1.3546754$

[31] Das A., Stöckelhuber K. W., Jurk R., Saphiannikova M., Fritzsche J., Lorenz H., Klüppel M., Heinrich G.: Modified and unmodified multiwalled carbon nanotubes in high performance solution-styrene-butadiene and butadiene rubber blends. Polymer, 49, 5276-5283 (2008).

DOI: 10.1016/j.polymer.2008.09.031

[32] Robertson C. G., Roland C. M.: Glass transition and interfacial segmental dynamics in polymer-particle composites. Rubber Chemistry and Technology, 81, 506522 (2008).

DOI: $10.5254 / 1.3548217$

[33] Bohm G. A., Tomaszewski W., Cole W., Hogan T.: Furthering the understanding of the non linear response of filler reinforced elastomers. Polymer, 51, $2057-$ 2068 (2010).

DOI: 10.1016/j.polymer.2010.01.047

[34] Heinrich G., Klüppel M.: Recent advances in the theory of filler networking in elastomers. Advances in Polymer Science, 160, 1-44 (2002). DOI: $10.1007 / 3-540-45362-81$

[35] Maier P. G., Goritz D.: Molecular interpretation of the Payne effect. Kautschuk Gummi Kunstoffe, 49, 18-21 (1996).

[36] Chazeau L., Brown J. D., Yanyo L. C., Sternstein S. S.: Modulus recovery kinetics and other insights into the payne effect for filled elastomers. Polymer Composites, 21, 202-222 (2000).

DOI: $10.1002 /$ pc. 10178
[37] Sternstein S. S., Zhu A-J.: Reinforcement mechanism of nanofilled polymer melts as elucidated by nonlinear viscoelastic behavior. Macromolecules, 35, 72627273 (2002). DOI: $10.1021 / \mathrm{ma} 020482 \mathrm{u}$

[38] Montes H., Lequeux F., Berriot J.: Influence of the glass transition temperature gradient on the nonlinear viscoelastic behavior in reinforced elastomers. Macromolecules, 36, 8107-8118 (2003).

DOI: $10.1021 / \mathrm{ma} 0344590$

[39] Zhu Z., Thompson T., Wang S-Q., von Meerwall E. D., Halasa A.: Investigating linear and nonlinear viscoelastic behavior using model silica-particle-filled polybutadiene. Macromolecules, 38, 8816-8824 (2005). DOI: $10.1021 / \mathrm{ma} 050922 \mathrm{~s}$

[40] Kalfus J., Jancar J.: Elastic response of nanocomposite poly(vinylacetate)-hydroxyapatite with varying particle shape. Polymer Composites, 28, 365-371 (2007). DOI: $10.1002 / p c .20273$

[41] Jancar J., Douglas J. F., Starr F. W., Kumar S. K., Cassagnau P., Lesser A. J., Sternsteinh S. S., Buehler M. J.: Current issues in research on structure-property relationships in polymer nanocomposites. Polymer, 51, 3321-3343 (2010).

DOI: $10.1016 /$ j.polymer.2010.04.074

[42] Funt J. M.: Dynamic testing and reinforcement of rubber. Rubber Chemistry and Technology, 61, 842-865 (1988).

DOI: $10.5254 / 1.3536222$

[43] Gauthier C., Reynaud E., Vassoille R., Ladouce-Stelandre L.: Analysis of the non-linear viscoelastic behaviour of silica filled styrene butadiene rubber. Polymer, 45, 2761-2771 (2004). DOI: $10.1016 /$ j.polymer.2003.12.081

[44] Huber G., Vilgis T. A.: Universal properties of filled rubbers: Mechanisms for reinforcement on different length scales. Kautschuk Gummi Kunstoffe, 52, 102107 (1999).

[45] Sternstein S. S., Ramorino G., Jang B., Zhu A-J.: Reinforcement and nonlinear viscoelasticity of polymer melts containing mixtures of nanofillers. Rubber Chemistry and Technology, 78, 258-270 (2005).

DOI: $10.5254 / 1.3547882$

[46] Galimberti M., Coombs M., Cipolletti V., Riccò T., Agnelli S., Pandini S.: The role of nanofillers in promoting hybrid filler networking and synergism with carbon black in a hydrocarbon rubber. Kautschuk Gummi Kunstoffe, 7-8, 31-36 (2013). 